\title{
Flt3-ITD mutations in a mouse model of radiation-induced acute myeloid leukaemia
}

Leukemia (2012) 26, 1445-1446; doi:10.1038/leu.2011.377; published online 6 January 2012

Acute myeloid leukaemia (AML) is one of the most common malignancies seen to occur in human populations exposed to ionising radiation. ${ }^{1}$ Mouse models have been widely used for quantitative and mechanistic studies of radiation leukaemogenesis; further, there is a similarity in the histopathological changes found in human AML and mouse AML. The majority of mouse radiation-induced AMLs (rAMLs) carry deletions of chromosome 2, and the Sfpi1/PU.1 haematopoietic transcription factor is located within the commonly deleted chromosome 2 region. ${ }^{2}$ In most rAMLs and rAML cell lines (70\%), Sfpi1/PU.1 suffers hemizygous loss accompanied by point mutations in the region encoding the ETS DNA-binding domain, leading to base substitutions at the R235 residue of the protein. ${ }^{2-4}$ Sfpi1/PU.1 mutations have been identified in human AML, but these are rare; in contrast, the most common mutations in human AML involve the Flt3 receptor tyrosine kinase. Most of these mutations are internal tandem duplication (Flt3-ITD) of $3-400 \mathrm{bp}^{5}$ that result in ligand-dependent dimerisation and receptor phosphorylation. The presence of a Flt3-ITD appears to confer a more severe phenotype and a poor prognosis for AML sufferers. The majority of the remaining non-ITD mutations in Flt3 occur in the second tyrosine kinase domain, being mostly point mutations within codon 835 or the deletion of $836^{6}$
In this study, for the first time, we identify Flt3 mutations in a murine model of $\mathrm{rAML}$, providing a direct mechanistic link between the mouse and human AML.

In all, 30 mouse rAML cases were screened for Flt3-ITD using PCR, 20 on an F1 CBA/H x C57BL/Lia background and 10 on an inbred $\mathrm{CBA} / \mathrm{H}$ background. All but nine of the $\mathrm{CBA} / \mathrm{H}$ and $\mathrm{rAMLs}$ have been described previously, ${ }^{2,4}$ the new AML samples were generated from mice irradiated with $3 \mathrm{~Gy}$ X-rays at MRC Harwell, Oxfordshire, UK. Animals were bred and maintained in accordance with the UK Animals (Scientific Procedures) Act 1986.

For each AML, DNA was extracted from leukaemic spleen tissue using a MagNA pure compact instrument (Roche Diagnostics $\mathrm{GmbH}$, Mannheim, Germany). Flt3-ITD mutations were detected using PCR with primers designed to amplify the region covering exons 14 and 15, syntenic to the region containing ITD in human FLT3 (F: $5^{\prime}-$ GCAATTAGGTACGAGAGTCAGC-3', R: $5^{\prime}$-CTITAGCATCTTCACCGC CACC-3'; Sigma-Aldrich, Poole, UK). The presence of FIt3-ITD was indicated by an increase in amplicon size and then confirmed by sequencing. Figure 1a provides a representative example of PCR analysis of events (Flt3-ITD) in mouse rAML. Flt3-ITDs, ranging in size from approximately 10 to $30 \mathrm{bp}$, were identified in three cases. Of these, two AMLs were heterozygous for the mutation and one homozygous. DNA sequencing of PCR products confirmed the presence of ITDs in these three AMLs: A, B and C of 18, 27 and $36 \mathrm{bp}$ in length, respectively, at independent insertion sites (Figure 1b).

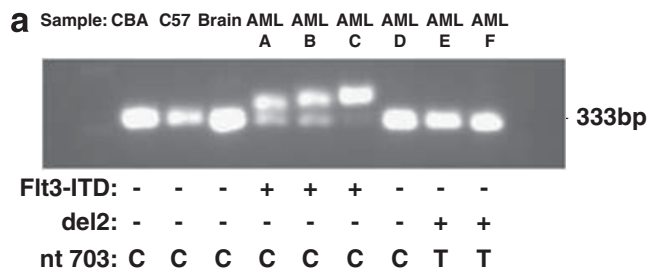

b

AMLB, nt 2022 acttctacgttgacttcagggactatg

CBA gagtacttctacgttgacttcaggaactatgaatatgaccttaagtgggagttcccgagagagaacttagagttt... nt 1990

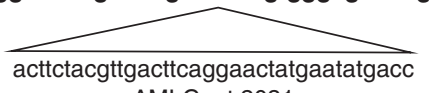
nt 2065 AMLC, nt 2031

CBA ..gccacggcctatggcattagtaaaacgggagtctcaattcaggtggcggtgaagatgctaaaaga nt 2220

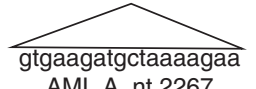

nt 2283 AML A, nt 2267

C AML B aa.598 DFYVDFKDY

CBA EYFYVDFRDYEYDLKWEFPRENLEFGKVLGSGAFGVMNATAYGISKYGVSIQVAVKMLK aa.588

$$
\begin{aligned}
& \text { HFYVDFRDYEY } \\
& \text { AML C aa.601D }
\end{aligned}
$$

Figure 1. (a) Representative Flt3-ITD PCR analysis of a panel of murine rAMLs run on a $2 \%$ agarose gel. The normal amplicon size is 333 bp. Presence of Flt3-ITD is indicated by an additional larger band on the gel. The gel is loaded as indicated above the image. 'CBA' and 'C57' refer to normal spleen DNA from CBA/H and C57BL/Lia, respectively; 'Brain' refers to brain tissue from the animal in which AML A developed and represents a normal tissue control; and AMLs A-F refer to independent AML samples. The genetic alterations identified in the AMLs are summarised below each sample (Flt3-ITD: + , presence and -, absence; del2: + , presence and - , absence; nucleotide 703 base at nucleotide 703 of Sfpi1/PU.1. C is wild type and T is mutant (the T allele leading to arginine 235 being converted to cysteine in Sfpi1/PU.1 protein). (b) CBA Flt3 Exon 14 and 15 sequence illustrating the three insertion sites and sequence of Flt3 ITD in AMLs A-C. ITD lengths for each are 18, 27 and $36 \mathrm{bp}$, respectively. (c) Predicted Flt3 protein sequence of AMLs A-C showing inserted amino acids. 
Table 1. Summary of the frequency of Flt3-ITD mutations, chromosome 2 deletions and Sfpi1/PU.1 R235 point mutations in a panel of 30 radiation-induced AMLs induced on two different genetic backgrounds

\begin{tabular}{lcccc}
\hline $\begin{array}{l}\text { AML genetic } \\
\text { background }\end{array}$ & Del2 & $\begin{array}{c}\text { Number } \\
\text { of samples }\end{array}$ & $\begin{array}{c}\text { Number with } \\
\text { Flt3-ITD (\%) }\end{array}$ & $\begin{array}{c}\text { Number with } \\
\text { Sfpi-1/PU.1 exon 5 } \\
\text { mutation (\%) }\end{array}$ \\
\hline $\mathrm{CBA} /$ & + & 17 & $0(0.0)$ & $14(82.4)$ \\
$\mathrm{H} \times \mathrm{C} 57 \mathrm{BL}$ & - & 3 & $2(66.7)$ & $0(0.0)$ \\
$\mathrm{CBA} / \mathrm{H}$ & + & 6 & $0(0.0)$ & $5(83.3)$ \\
& - & 4 & $1(25)$ & $0(0.0)$ \\
\hline Abbreviations: AML, acute myeloid leukaemia; Del2, chromosome 2 deletion.
\end{tabular}

These ITDs were predicted by BLAST to lead to 6-12 novel amino acids being inserted in frame (Figure 1c).

The majority of rAMLs in this study $(23 / 30,77 \%)$ carry chromosome 2 deletions as identified by loss-of-heterozygosity or in situ hybridisation methods. ${ }^{2,7}$ Of these, most $(19 / 23,82 \%)$ carry Sfpi1/PU.1 mutations affecting R235 (Table 1). However, all Flt3-ITD occurred in rAMLs in which no Sfpi1/PU.1 involvement was identified, either as chromosome 2 deletions or as Sfpi1/PU.1 exon 5 mutations. Out of seven rAMLs without Sfpi1/PU.1 alterations, three have presented with Flt3-ITDs ( 43\%). To check for the presence of small Flt3-ITD mutations in those AMLs that were assessed as negative for the ITD mutations using PCR and gelbased assays, Flt3 exon 14/15 PCR products from a sample of 11 AMLs $(9$ from $\mathrm{CBA} / \mathrm{H}$ animals and 2 from CBA $\times$ C57BL/6Lia animals; 8 with chromosome 2 deletions and 3 without chromosome 2 deletions) were sequenced. None of these was found to have any exon 14/15 Flt3 DNA sequence alterations. Therefore, no ITDs were found in any of these rAMLs with chromosome 2 deletions (with or without Sfpi1/PU.1 exon 5 mutations). Statistical analysis using Fisher's exact test to compare Flt3-ITD and chromosome 2 deletion status indicated that the absence of AMLs with both Flt3-ITD and chromosome 2 deletions was statistically significant $(P=0.0086)$. These findings suggest that the involvement of Flt3-ITD and Sfpi1/PU.1 is mutually exclusive in this model. No phenotypic differences between AMLs with the involvement of Flt3-ITD and Sfpi1/PU.1 have been observed.

To rule out the possibility of point mutations within the second tyrosine kinase domain of Flt3, we screened samples that showed no Sfpi1/PU.1 involvement using PCR with primers covering exon 20 syntenic to the mutated region in human $A M L$ (F: $5^{\prime}-A G A$ AGAGGCTGGCAGAAGAA-3', R: $5^{\prime}$-CCGTAGGACCAGACGTCACT-3'). Fragments were sequenced along with corresponding matched brains (negative controls). No cases of point mutations were identified in any of the rAMLs, indicating that Flt3-ITD is the only Flt3 mutation present in these rAMLs.

This study has therefore identified Flt3-ITD mutations in approximately $10 \%$ of mouse rAMLs for the first time, but only in cases without Sfpi1/PU.1 involvement. The Flt3-ITDs are in the size range identified in humans ${ }^{6}$ and although rare in our samples, show greater parallels to human AML. The three insertions are predicted to lead to 6-12 novel amino acids being inserted in frame in Flt3 with two of these insertions in the tyrosine-rich juxtamembrane domain; these ITDs are therefore similar to those seen in human AML cases. ${ }^{8}$ The findings also suggest that radiation can cause Flt3-ITD mutations directly.

There are therefore two identified pathways leading to rAML in the mouse, the most common involving loss/mutation of Sfpi1/PU.1 and the other involving mutation of Flt3. Future work will help establish the mechanistic relationship between the mouse rAMLs and the human disease. The Flt3-ITD-carrying AMLs, although rare, may be of value in the preclinical evaluation of FLT3 inhibitors as therapeutic agents for myeloid leukaemia.

\section{CONFLICT OF INTEREST}

The authors declare no conflict of interest.

\section{ACKNOWLEDGEMENTS}

We thank Kevin Whitehill, Margaret Coster, Pat Hillier and Donna Lowe for assistance with mouse studies and Liz Ainsbury for statistical advice. This work was supported by the US Department of Energy grant DE-FG02-05ER63947, the European Commission RISC-RAD project, F16R-CT-2003-508842 and the National Institute for Health Research Centre for Research in Health Protection Research at the Health Protection Agency.

\section{DISCLAIMER}

This report is in part work commissioned by the National Institute for Health Research. The views expressed in this publication are those of the authors and not necessarily those of the NHS, the National Institute for Health Research or Department of Health.

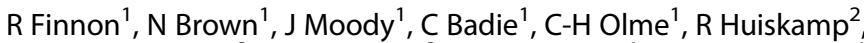
E Meijne $^{3}$, M Sutmuller $^{2}$, M Rosemann $^{4}$ and SD Bouffler ${ }^{1}$

${ }^{1}$ Biological Effects Department, Health Protection Agency, Centre for Radiation, Chemical and Environmental Hazards, Oxon, UK

${ }^{2} N R G$, Petten, The Netherlands,

${ }^{3}$ Universitair Medisch Centrum Groningen (UMCG), Groningen, The Netherlands and

${ }^{4}$ Helmholtz Zentrum Muenchen, Institute for Radiation Biology, Muenchen, Germany E-mail: simon.bouffler@hpa.org.uk

\section{REFERENCES}

1 United Nations. Sources, Effects and Risks of lonizing Radiation, United Nations Scientific Committee on the Effects of Atomic Radiation. 2006 Report to the General Assembly, Annex A. United Nationals: New York, 2008.

2 Silver A, Moody J, Dunford R, Clark D, Ganz S, Bulman R et al. Molecular mapping of chromosome 2 deletions in murine radiation-induced AML localizes a putative tumour suppressor gene to a $1.0 \mathrm{cM}$ region homologous to human chromosome segment 11p11-12. Genes Chromosomes Cancer 1999; 24: 95-104.

3 Suraweera N, Meijne E, Moody J, Carvajal-Carmona L, Yoshida K, Pollard P et al. Mutations of the PU.1 etc domain are specifically associated with murine radiationinduced, but not human therapy-related acute myeloid leukaemia. Oncogene 2005; 24: $3678-3683$.

4 Brown NL, Finnon R, Bulman RA, Finnon P, Moody J, Bouffler SD et al. Sfpi1/PU.1 mutations in mouse radiation-induced acute myeloid leukaemias affect mRNA and protein abundance and associate with disrupted transcription. Leuk Res 2011; 35: $126-132$.

5 Gilliland G, Griffin J. The roles of Flt3 in hematopoiesis and leukaemia. Blood 2002 100: $1532-1542$.

6 Small D. Flt3 mutations: biology and treatment. Hematology Am Soc Hematol Educ Program 2006, 178-184. Review.

7 Peng Y, Brown N, Finnon R, Warner CL, Liu X, Genik PC et al. Radiation leukaemogenesis in mice: loss of PU.1 on chromosome 2 in CBA and C57BL/6 mice after irradiation with $1 \mathrm{GeV} /$ nucleon ${ }^{56} \mathrm{Fe}$ ions, $\mathrm{x}$ rays or gamma rays. Part 1 . Experimental Observations. Radiat Res 2009; 171: 474-483.

8 Kiyoi H, Towatari M, Yokota S, Hamaguchi M, Ohno R, Saito H et al. Internal tandem duplication of the Flt3 gene is a novel modality of enlongation mutation which causes constitutive activation of the product. Leukaemia 1998; 12: 1333-1337. NonCommercial-No Derivative Works 3.0 Unported License. To view a copy of this license, visit http://creativecommons.org/licenses/by-nc-nd/3.0/ 